Document downloaded from:

http://hdl.handle.net/10251/57369

This paper must be cited as:

Barceló Rico, F.; Gozálvez Zafrilla, JM.; Diez Ruano, JL.; Santafé Moros, MA. (2011). Modelling and control of a continuous distillation tower through fuzzy techniques. Chemical Engineering Research and Design. 89(1):107-115. doi:10.1016/j.cherd.2010.04.015.

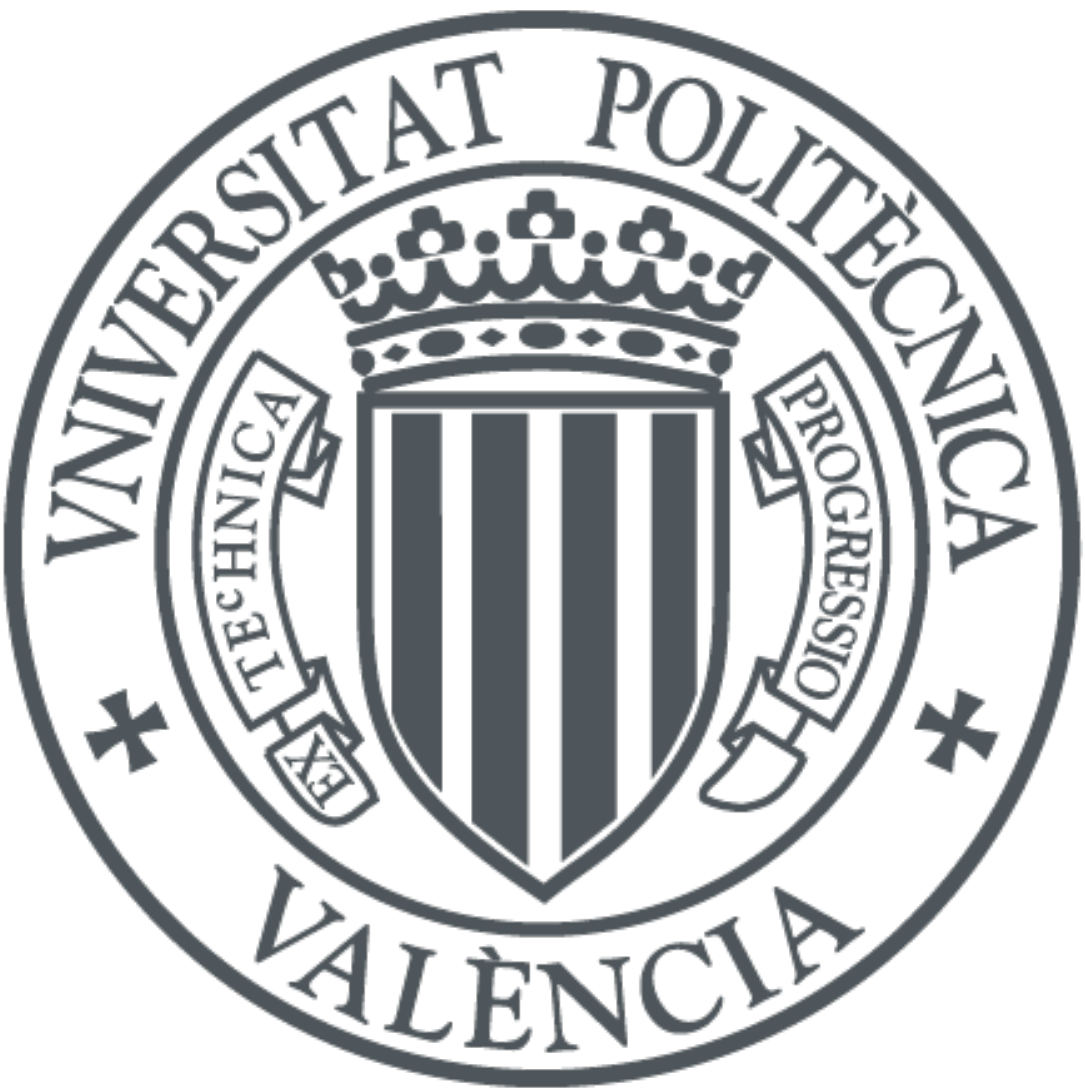

The final publication is available at

http://dx.doi.org/10.1016/j.cherd.2010.04.015

Copyright Elsevier

Additional Information 


\title{
Modelling, control and optimization of a continuous distillation tower through fuzzy techniques
}

\author{
Fátima Barceló-Rico*, José M. Gozálvez-Zafrilla**, José Luis Díez*, and \\ Asunción Santafé-Moros** \\ *Department of Systems Engineering and Control \\ *Department of Chemical and Nuclear Engineering \\ Universidad Politécnica de Valencia \\ Camino de Vera, 14 \\ 46022 Valencia, Spain \\ E-mail: fabarri@upv.com,jmgz@iqn.upv.es
}

\begin{abstract}
This paper presents a methodology for the design of a fuzzy controller applicable to continuous processes based on local fuzzy models and velocity linearizations. It has been applied to the implementation of a fuzzy controller for a continuous distillation tower. Continuous distillation towers can be subjected to variations in feed characteristics that cause loss of product quality or excessive energy consumption. Therefore, the use of a fuzzy controller is interesting to control process performance.

A dynamic model for continuous distillation was implemented and used to obtain data to develop the fuzzy controller at different operating points. The fuzzy controller was built by integration of linear controllers obtained for each linearization of the system. Simulation of the model with controller was used to validate the controller effectiveness under different scenarios.

The results showed that the fuzzy controller was able to keep the target output in the desired range for different inputs disturbances, changing smoothly from a predefined target output to another. The developed techniques are applicable to more complex distillation systems including more operating variables.
\end{abstract}

Key words: fuzzy, distillation tower, gain scheduling, control 


\section{Introduction.}

This paper presents a methodology for the design of a fuzzy controller applicable to continuous processes. The controller is based on a fuzzy model describing the plant dynamics that is previously calculated using experimental or model data. In this case, the technique has been applied to the control of a continuous distillation tower.

Continuous distillation is one of the most used separation processes in the processing of large amount of products. This makes it a highly energyconsuming operation [12]. Usually, distillation towers are designed for a feed stream of constant characteristics and classic control (PI or PID controllers) is used to change the operation parameters in order to achieve the specifications required for the products. However, there are some problems that make this approach inefficient: Distillation control is difficult because of its usually nonlinear, non-stationary, interactive, and is subject to constraints and disturbances [10]. For example, feed streams can be subjected to important variations in flow, composition or energy state. Therefore, to control the top and bottom compositions of a distillation column can be a difficult task due to the presence of control-loop interactions and nonlinearities [6].

An efficient control system adaptable to different situations would assure product quality and minimize energy expenses [9], [8].

New approaches to control, other than classic linear controllers, can improve system performance. The most common of the advanced approaches is the use of nonlinear multivariable models. However, the nonlinear models tend to become rigorous and computationally intensive as the process behavior becomes more complex [6]. Other approaches, like control based in neural networks, have interesting properties, like generality of model structure, capacity of learning from experimental data and expressing the process non-linearities and calculation speed [5]. Control based on fuzzy modelling is an alternative to neural network based control that also shows some of these characteristics. Fuzzy control has been satisfactorily applied to many complex systems of different fields of science and engineering characterized by significant nonlinearities and/or noise. Any static or dynamic system that makes use of fuzzy sets is called a fuzzy system. In addition to their universal function approximation capabilities, fuzzy models resemble human reasoning processes, providing the readability of the obtained representations [13]. Fuzzy models can therefore be validated by experts and incorporate additional qualitative or imprecise information that engineers or operators may 
have about the system.

A fuzzy model is usually automatically developed from experimental data by a rule extraction method based on genetic algorithms, neural networks, templates or clustering techniques [1], [3]. However, in the case of distillation, the results provided by dynamic models are very accurate and can be used instead of experimental data. Therefore, a grey box approach, combining basic knowledge about the system and black box models, has been considered more suitable for this work. The final model built in this way consists of a set of local models (one for each rule) at different operating points, and it avoids fuzzy interpolation problems by means of velocity-based linearization [7], [2].

The second section of this report briefly describes the equations of the dynamic distillation model and their implementation. The third and fourth sections present the theory of the fuzzy controller and the methodology used to build it for an example case. The fifth section shows the criteria used to choose the optimum variable to perform the control. Finally, in the last section, a discussion of the performance of the fuzzy controller for the example case is presented.

\section{Dynamic Model for Continuous distillation}

Distillation towers are multi-stage separation units conceived to separate feeds into streams enriched in components of different volatility. The separation is achieved when a vapour and a liquid stream tend to thermodynamic equilibrium when are put in contact in the separation stages (trays).

Figure 1 shows a schematic representation of the tower and the streams flows between its different stages $i$. The stages are numbered increasingly from top to bottom: 1 is the condenser, 2 to $\mathrm{N}-1$ are the trays and $\mathrm{N}$ is the reboiler. Every stream is defined by their flow, molar fractions of $j$ components and enthalpy. Thus, a feed entering to stage $\mathrm{i}$ is defined by $F_{i}, z_{i, j}$ and $h_{F i}$. The liquid and vapour streams exiting from the stage $i$ are defined by $L_{i}, x_{i j}, h_{L i}$ and $G_{i}, y_{i, j}, h_{G i}$ respectively. A duty heat, $q_{N}$, is supplied to the reboiler in order to generate the vapour stream. The vapour stream moves upwards exchanging components with the descending liquid stream, and the vapour is progressively enriched in the most volatile components. Consequently, the liquid stream will be enriched in the less volatile ones. To generate a saturated liquid stream, a heat duty $q_{1}$ must be removed from the vapour in the condenser. This stream is divided into a 
product stream and a stream recycled back to the column. The recirculation is determined by the reflux ratio, $R$, defined in (1), which constitutes one of the main operation variables.

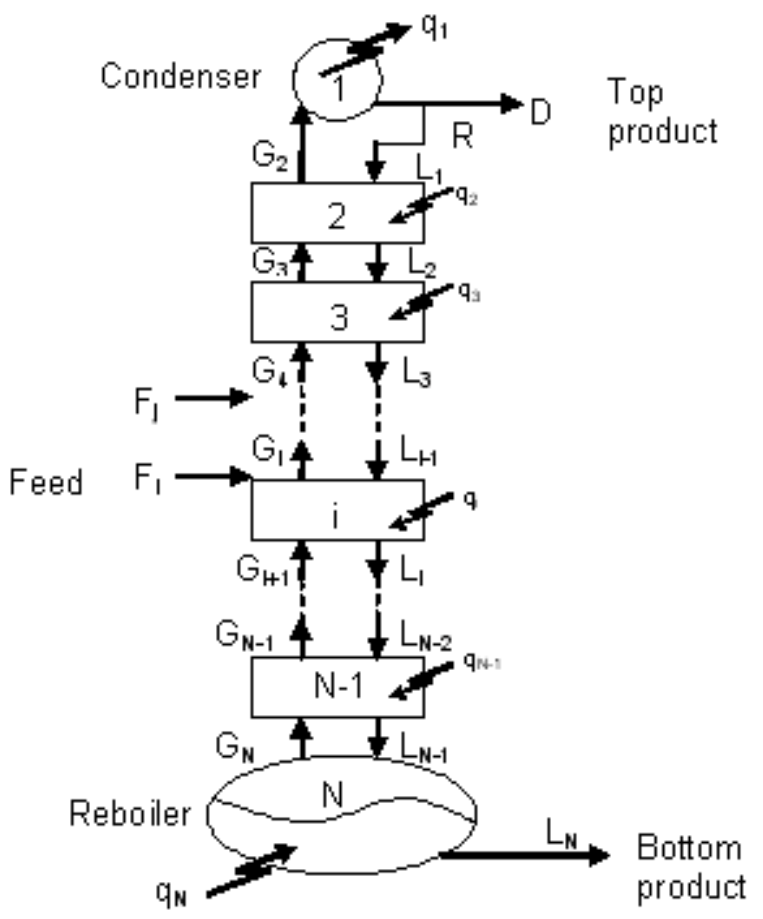

Figure 1: Scheme of the continuous distillation tower from basic principles

$$
R=\frac{L_{1}}{D}
$$

For every stage $i$, non-stationary balances of total amount of moles $M_{i}$, moles of each component $j$ and energy can be established (2-4). These equations constitute the basis to obtain the dynamic model of the system [12], $[11]$.

$$
\begin{gathered}
\frac{d M_{i}}{d t}=L_{i-1}-L_{i}+G_{i+1}-G_{i}+F_{i} \\
\frac{d\left(M_{i} \cdot x_{i, j}\right)}{d t}=L_{i-1} \cdot x_{i-1, j}-L_{i} \cdot x_{i, j}+G_{i+1} \cdot y_{i+1, j}-G_{i} \cdot y_{i, j}+F_{i} \cdot z_{i, j}
\end{gathered}
$$




$$
\frac{d\left(M_{i} \cdot h_{L i}\right)}{d t}=L_{i-1} \cdot h_{L i-1}-L_{i} \cdot h_{L i}+G_{i+1} \cdot h_{G i+1}-G_{i} \cdot h_{G i}+F_{i} \cdot h_{F i}+q_{i}
$$

Substitution of (2) into (3) and (4) leads to the time derivatives of composition and enthalpy $(5,6)$.

$$
\begin{gathered}
\frac{d x_{i, j}}{d t}=M_{i}^{-1} \cdot\left[\begin{array}{c}
L_{i-1} \cdot x_{i-1, j}+F_{i} \cdot z_{F, i}+G_{i+1, j} \cdot y_{i+1, j}+ \\
+\left(G_{i, j}-G_{i+1, j}-L_{i-1}-F_{i}\right) \cdot x_{i, j}-G_{i, j} \cdot y_{i, j}
\end{array}\right] \\
\frac{d h_{L, i}}{d t}=M_{i}^{-1} \cdot\left[\begin{array}{l}
L_{i-1} \cdot h_{L, i-1}+F_{i} \cdot h_{F, i}-L_{i} \cdot h_{L, i}+G_{i+1} \cdot h_{G, i+1}+q_{i}- \\
-G_{i} \cdot h_{G, i}+h_{L, i} \cdot\left(L_{i-1}+F_{i-1}-L_{i}+G_{i-1}-G_{i}\right)
\end{array}\right]
\end{gathered}
$$

Assuming that there is enough contact time in each stage, the exiting phases will be close to equilibrium. So, using a thermodynamic model for the mixture, the vapour composition can be expressed as a function of the liquid composition. Besides, for a saturated liquid, the enthalpy and temperature depend exclusively on the liquid composition, hence we can express:

$$
\frac{d h_{L i}}{d t}=\left.\sum_{j} \frac{\partial h_{L}}{\partial x_{j}}\right|_{x_{i, j}} \cdot \frac{d x_{i, j}}{d t}
$$

Our aim is to relate the stream flows and the molar fractions in the liquid that remain as the only state-variables of the problem. To have a unique vector $\mathrm{W}$ representing all the unknown internal flows, an index operator $\mathrm{n}(\mathrm{i}, \mathrm{k})$ is defined for each stage $i$ so that for indices $\mathrm{k}=0$ to 4 stand for the streams $F_{i}, G_{i}, L_{i-1}, G_{i+1}$ and $L_{i}$ respectively.

$$
n(i, k)=4 \cdot(i-1)+k
$$

Additionally, an operator sign $(\mathrm{k})$ is defined as $(-1)$ for the output streams and $(+1)$ for the input ones. Thus, combination of equations (5-7) yields to:

$$
\sum_{k=1}^{3} a_{n(i, k)}^{e} \cdot W_{n(i, k)}=q_{i}-a_{n(i, 0)}^{e} \cdot W_{n(i, 0)}
$$

where, 


$$
a_{n(i, k)}^{e}=h_{n(i, 4)}-h_{n(i, k)}+\operatorname{sign}(k) \sum_{j}\left[\left.\frac{\partial h_{L}}{\partial x_{j}}\right|_{x_{i, j}} \cdot\left(x_{n(i, k), j}-x_{i, j}\right)\right]
$$

Additional equations are necessary to completely determine the stream flows. These equations can be obtained by expressing the liquid density as a function of the liquid composition and considering constant volume of liquid $V_{i}$ as the liquid overflows in each stage:

$$
\frac{d M_{i}}{d t}=V_{i} \frac{d \rho}{d t}=\left.V_{i} \sum_{j} \frac{\partial \rho}{\partial x_{j}}\right|_{x_{i, j}} \cdot \frac{d x_{i, j}}{d t}
$$

Thus, the latter equation leads to following relationship:

$$
\sum_{k=1}^{4} a_{n(i, k)}^{v} \cdot W_{n(i, k)}=-a_{n(0, k)}^{v} \cdot W_{i, 0}
$$

where,

$$
a_{n(i, k)}^{v}=-1+\frac{\operatorname{sign}(k)}{\rho\left(\vec{x}_{n(i, 4)}\right)} \sum_{j}\left[\left.\left(x_{n(i, k), j}-x_{i, j}\right) \frac{\partial \rho}{\partial x_{j}}\right|_{x_{i, j}}\right]
$$

Equations (9) and (12) constitute a linear system to solve the unknown internal flows as a function of the liquid composition. The coefficients of the system can be rearranged as a banded matrix. As this matrix is usually badconditioned, the Moore-Penrose pseudoinverse was used to solve the system of equations.

Therefore, the set of differential equations (5) can now be solved to obtain the evolution of the state-variables of the system. A multi-step stiff solver (Adams-Bashforth-Moulton PECE) was used to compute the tower dynamics as a function of feed, $F_{i}$, reflux ratio, $R$, and reboiler heat duty, $q_{N}$.

\section{Velocity-Based Fuzzy Control of a Continuous Distillation Pro- cess}

A suitable modelling and identification of a system is essential for controller design. In our case, fuzzy identification adjusts those models to available data sets, and the local error of a number of local models that represent 
the system in a region is preferred to the usual approach of minimizing the global prediction error [4].

The identification of fuzzy models needs to establish a model structure for a subsequent parameter identification step [13], as occurs in all identification procedures. This second identification step can be easily done, for example, by least mean squares if the system is linear in parameters [1]. This is our case, because input and output variables, and antecedent fuzzy membership functions A (interpretable as validity regions for local descriptions in a set of operating points) will be provided by experts, and rule consequents will be affine models following Takagi-Sugeno structure described by:

$$
\begin{aligned}
& R_{i}: \text { If }\left(x \text { is } A_{i}\right) \text { then }\left(y_{i}=a_{i}^{T} \cdot x+b_{i}\right) \\
& i=1,2, \ldots, k
\end{aligned}
$$

where $a_{i}$ is a vector of parameters, and $b_{i}$ is a scalar. The model output $y$ will be the convex combination of the consequents by means of its membership functions $\mu_{i}$ :

$$
y=\frac{\sum_{i=1}^{k} \mu_{i}(x) \cdot y_{i}}{\sum_{i=1}^{k} \mu_{i}(x)}
$$

Although the fuzzy model structure showed in (14) and (15) can give accurate results for prediction purposes, when the final goal is process control, a better approach overcoming interpolation problems caused by the terms $b_{i}$ is the use of velocity-based models [7], [2]. These result from the substitution of (14) by its incremental form (16) where offset term is avoided.

$$
\begin{aligned}
& R_{i}: \text { If }\left(\Delta x \text { is } A_{i}\right) \text { then }\left(\Delta y_{i}=\Delta a_{i}^{T} \cdot \Delta x\right) \\
& i=1,2, \ldots, k
\end{aligned}
$$

A controller will be then designed for each rule, leading to the modelling and control structures outlined in Figures 2 and 3.

In order to give theoretical framework for the proposed controller design technique, it is going to be applied as it was a classic gain scheduling controller [7]. A possible approach to gain scheduling requires following the subsequent steps [2]: 


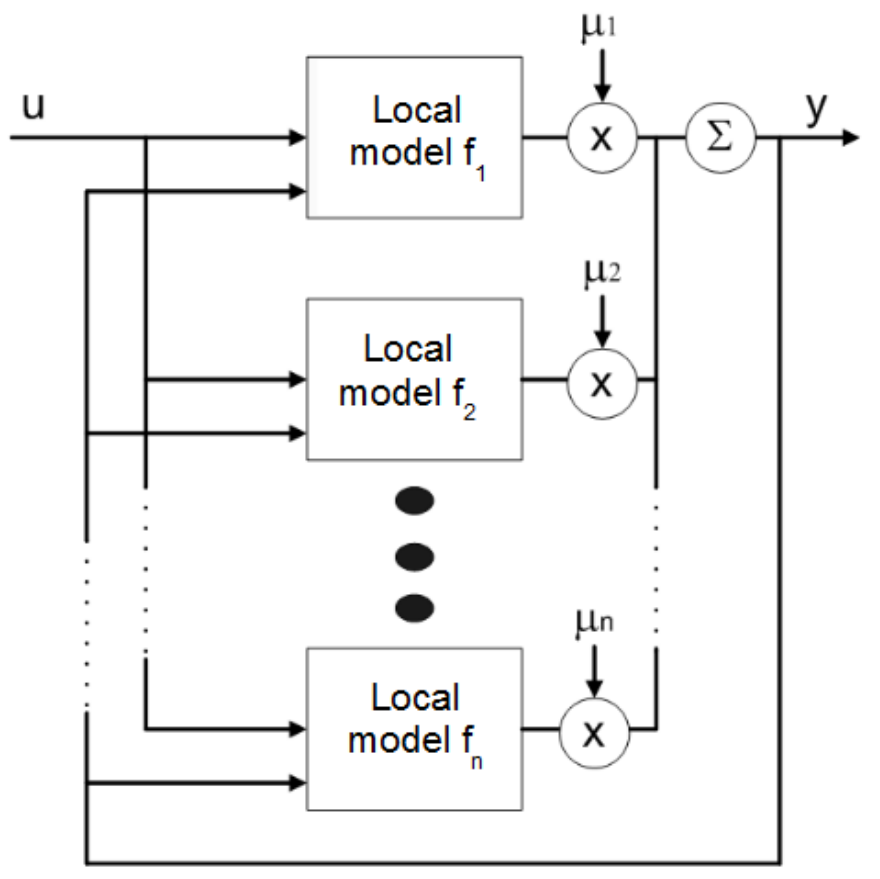

Figure 2: Outline of suggested structure for modelling

1. Determine a Linear Parameter Varying model of the non-linear system based on the linearization of the system at different operating points and integrating them, for example, by interpolation.

2. Calculate a Linear Controller for each linearization of the system and then integrate the controllers (for example, in the same way as the linearization), then obtaining a Non-Linear Controller.

3. Evaluate the control performance through simulation.

In our case, the proposal is that, once the operating points are given by the expert, a fuzzy model in the form of (15) with rules as (16) will be tuned using experimental data. A model defined with this structure is a combination of purely linear (not affine) models, then matching the first step in the gain scheduling approach.

Next step will be the design of a linear (using classic techniques) controller for each rule consequent (i.e, each linearization or local model). The combi- 


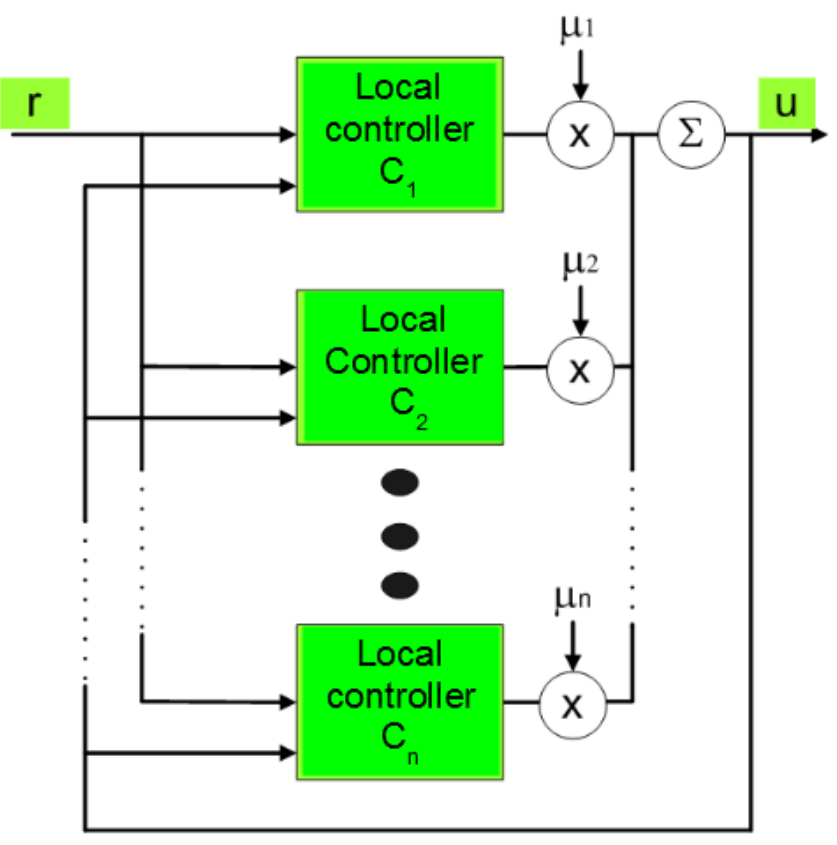

Figure 3: Outline of suggested structure for control

nation of all the controllers will be done using the same formula (15) used in the fuzzy modelling, but substituting local model output by local control action. In this way, a non-linear controller is designed as in the second step of the gain scheduling approach.

Performance can be tested via simulations, and a complete example is presented in the next section.

\section{Fuzzy-Model Results and Validation}

The control system can be built using data from a real system. However, dynamic distillation models are accurate enough and can be used to generate data avoiding experimental cost. As an application example, a dynamic distillation model as described in section 2 has been used to obtain the necessary data to implement the fuzzy controller for a distillation of a mixture methanol-water. Parameters of the column are summarized in table 
Table 1: Characteristics of the distillation column

\begin{tabular}{|l|c|c|}
\hline Parameter & Nomenclature & Value (Unities) \\
\hline Number of trays & $\mathrm{N}$ & 10 \\
\hline Feed tray position & $F_{i}$ & 6 \\
\hline Tray volumen & $V_{i}$ & $0.064\left(\mathrm{~m}^{3}\right)$ \\
\hline Condenser volumen & $V_{C}$ & $0.4\left(\mathrm{~m}^{3}\right)$ \\
\hline Reboiler volumen & $V_{R}$ & $0.4\left(\mathrm{~m}^{3}\right)$ \\
\hline
\end{tabular}

Table 2: Inputs (steps) applied to the simulator

\begin{tabular}{|l|c|c|c|}
\hline Input & Nomenclature & Small step & Big Step \\
\hline Heat of reboiler & $q_{N}$ & $\pm 7.5 \%$ & $\pm 15 \%$ \\
\hline Enthalpy & $h_{F}$ & $\pm 7.5 \%$ & $\pm 15 \%$ \\
\hline Reflux & $R$ & $\pm 2.5 \%$ & $\pm 5 \%$ \\
\hline
\end{tabular}

(1). Both the model and the simulator (next section) where used to test the capability of the fuzzy controller under a set of scenarios.

The input disturbances applied are summarized in table (2). It can be seen that the changes (in percentatge) applied to $q$ and $h_{F}$ are the same, while for $R$ are smaller. This is due to the fact that bigger changes made the system unstable, as this in an input that affects very much to the composition of the products of the process. The analysis of the results by experts yielded to the following conclusions:

- a positive increment in the inputs $F$ and $z_{F}$ causes a positive increment on $x_{10}$,

- a positive increment in $h_{F}, q_{N}$, and $\mathrm{R}$ causes a negative increment on $x_{10}$,

- a positive increment in $z_{F}$ and $q_{N}$ causes a positive increment on $x_{1}$,

- a positive increments in $F, h_{F}$ and $\mathrm{R}$ causes a negative increment on $x_{1}$

- the system response is similar to a first order system with a time constant of the system for each input at each working point for each output variable, and 
Table 3: Operating points for the system under study

\begin{tabular}{|c|c|c|c|c|c|}
\hline Point & $F\left(k m o l * s^{-1}\right)$ & $z_{F}$ & $h_{F}\left(k J * k m o l^{-1}\right)$ & $q_{N}\left(k J * s^{-1}\right)$ & $R$ \\
\hline P1 & 0.217 & 0.36 & 11000 & 1878 & 1 \\
\hline P2 & 0.217 & 0.46 & 11000 & 1878 & 1 \\
\hline P3 & 0.217 & 0.26 & 11000 & 1878 & 1 \\
\hline P4 & 0.217 & 0.36 & 20000 & 1878 & 1 \\
\hline P5 & 0.217 & 0.36 & 11000 & 2744 & 1 \\
\hline P6 & 0.217 & 0.36 & 11000 & 1100 & 1 \\
\hline
\end{tabular}

- a set of six operating points (defined as combinations of $F, z_{F}, h_{F}, q_{N}$, and $R$ ) are the most common for system operation, and are summarized in Table 3.

Then, a set of 5 models (one for each input/output pair) for each point (and then 30 systems for each output) was developed. As far as $F$ and $z_{F}$ are usually constant and that the most important output of the system is $x_{1}$, (and the control of product composition $x_{1}$ will modify $x_{10}$ ), a simplified input-output view (including possible control variables as inputs) of these small models is devised in Figure 4. This set of first order systems will be integrated with the fuzzy structure proposed in the previous section.

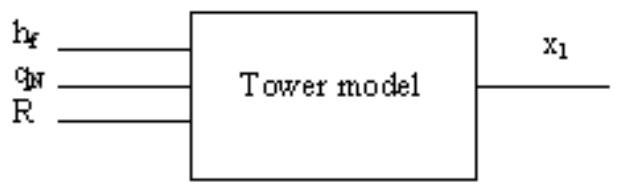

Figure 4: Scheme of the continuous distillation tower from simple black box model point of view

Equation (17) shows the G general single input single output linear discrete first order system (input $u$, output $y$, and instant $k$ ) placed at the consequent of each rule:

$$
G=\frac{k}{z-\tau} \rightarrow y_{k}=\tau \cdot y_{k-1}+k \cdot u_{k}
$$


That will be used in its incremental form:

$$
\Delta y_{k}=\tau \cdot \Delta y_{k-1}+k \cdot \Delta u_{k}
$$

Therefore, the fuzzy system considered 5 inputs $\left(F, z_{F}, h_{F}, q_{N}, R\right)$ in order to indicate the operating point in what the tower is working, and 5 more inputs $\left(F, z_{F}, h_{F}, q_{N}, R\right)$ to indicate the increment that it was applied to each input, and finally one more input to indicate the previous output $\left(y_{k-1}\right)$. Rule selection was carried out for the first five inputs, and the other ones were used to calculate the value of the output. As the systems in this case just depend on one input, the coefficients for the others are zero. The behaviour obtained for the models was very good for the whole operation systems, being the modelling error lower than $5 \times 10^{-5 \%}$. As an example, the model performance around the operating point 5 is shown in Figure 5, but results are similar for the whole operating space.

Once the model was available, the controller could be designed. In this case it was a simple pole-zero for each rule consequent, following for its combination the structure of Figure 4. In fact, one controller was designed for each submodel and then the total model was built for all the controllers joined in a fuzzy system. The general incremental control equation is:

$$
\Delta u_{k}=\frac{(p-\tau)}{k} \cdot \Delta y_{k}+\frac{(1-p)}{k} \cdot \Delta r_{k}
$$

where $p$ is the chosen pole.

From a practical point of view, the controller can be applied to keep the target output in the desired range if unexpected disturbances occur in the feed. The controller must also be applied to set $x_{1}$ at a value of interest, and changed it to another one if necessary. It can be reached operating on one of these: $q_{N}, h_{F}$ or $R$. System feedback response is shown in Figures 6,7 and 8 in several operating points (Table 4 ). The reference is set in percentage and is the same for each operating variable in all points. It is important to select a reference which is within the control boundaries of the operating variables.s

Figures 6,7 and 8 show how the control by different variables leads to very good results in all cases in spite of the different characteristics of the controlled variable. The settling time is different for each of them as well as the dynamics of the controlled signal $\left(x_{1}\right)$, but for a control variable all the controlled signals are similar, even when the operating points are very different. Besides, all control variables perform accurately enough even in 

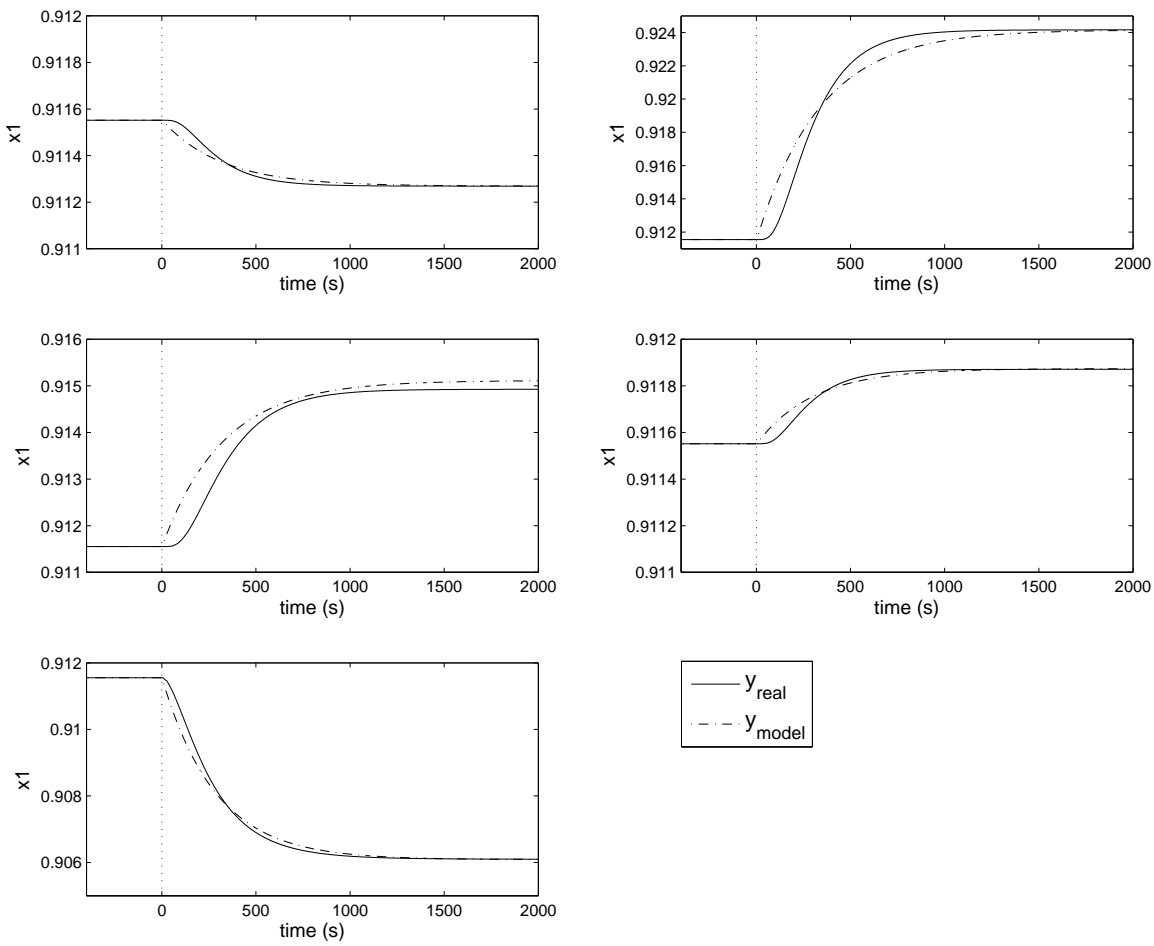

Figure 5: Models of molar fraction $x_{1}$ versus time (in seconds) at the operating point 5 . From top left to bottom right, variations of $x_{1}$ for disturbances in $F, z_{F}, h_{F}, q$, and $R$.

points that are not exactly the operating points for which the controllers were designed. This is a good property of this type of controllers.

The advantage of having different control variables meeting the same requirements is that its effectiveness can be checked from different points of view (for example: economical, resources availability, etc.) and the decision on what control variable must be used can change from time to time depending on external factors. 
Table 4: Operating points where the control has been applied

\begin{tabular}{|c|c|c|c|c|c|}
\hline Point & $F\left(k m o l * s^{-1}\right)$ & $z_{F}$ & $h_{F}\left(k J * k m o l^{-1}\right)$ & $q_{N}\left(k J * s^{-1}\right)$ & $R$ \\
\hline OP1 & 0.217 & 0.43 & 15000 & 2079 & 1 \\
\hline OP2 & 0.217 & 0.32 & 12000 & 2426 & 1 \\
\hline OP3 & 0.217 & 0.28 & 19000 & 1473 & 1 \\
\hline OP4 & 0.217 & 0.36 & 14000 & 1600 & 1 \\
\hline
\end{tabular}

\section{Control applied to physical model}

Once the set of models has been built and the set of controllers tested with the models and proved their performance, the next step is to apply the set of fuzzy controllers to the simulator of the plant, as this has a slightly different behaviour of the model built and therefore, the performance of the controller might be different over the simulator than over the model.

To apply the controller to the simulator it has to be taken into account that the output and inputs for this are absolute and not by increments as they were for the model (Velocity-based model and controller, equation 16).

Figure 9 shows the control applied to the simulator in operating point $P C 1=\left[\begin{array}{lllll}0.217 & 0.36 & 15000 & 1878 & 1\end{array}\right]$, which is a point between P1 and $\mathrm{P} 4$. It can be seen how the control performs very well in spite of the speed and dynamics of the controlled signal $x_{1}$.

It has been said that the speed of feeding is constant as well as the composition of this feeding in this kind of processes. Nevertheless, it can happen that the composition has some perturbations due to small impurities of the product. This can highly modify the effects of the control. Thus, to check the performance of the fuzzy controller when perturbations are present in the composition different variations in composition $C_{o}$ have been introduced. Figure 10 shows how the control makes the signal come back to the reference, even when a perturbation of $10 \%$ is present in $C_{o}$.

It has to be said that control variables $h_{F}$ and $q_{N}$ are not suitable to control the composition of $x_{1}$ when big perturbations are present in $C_{o}$. They can only control up to perturbations of $0.1 \%$. This is due to dynamics of the composition of the products when this variables are modified. On the other hand, the modification of the variable $R$ has an immediate response on the output composition. This makes possible the control of $x_{1}$ even when big perturbations are present. 

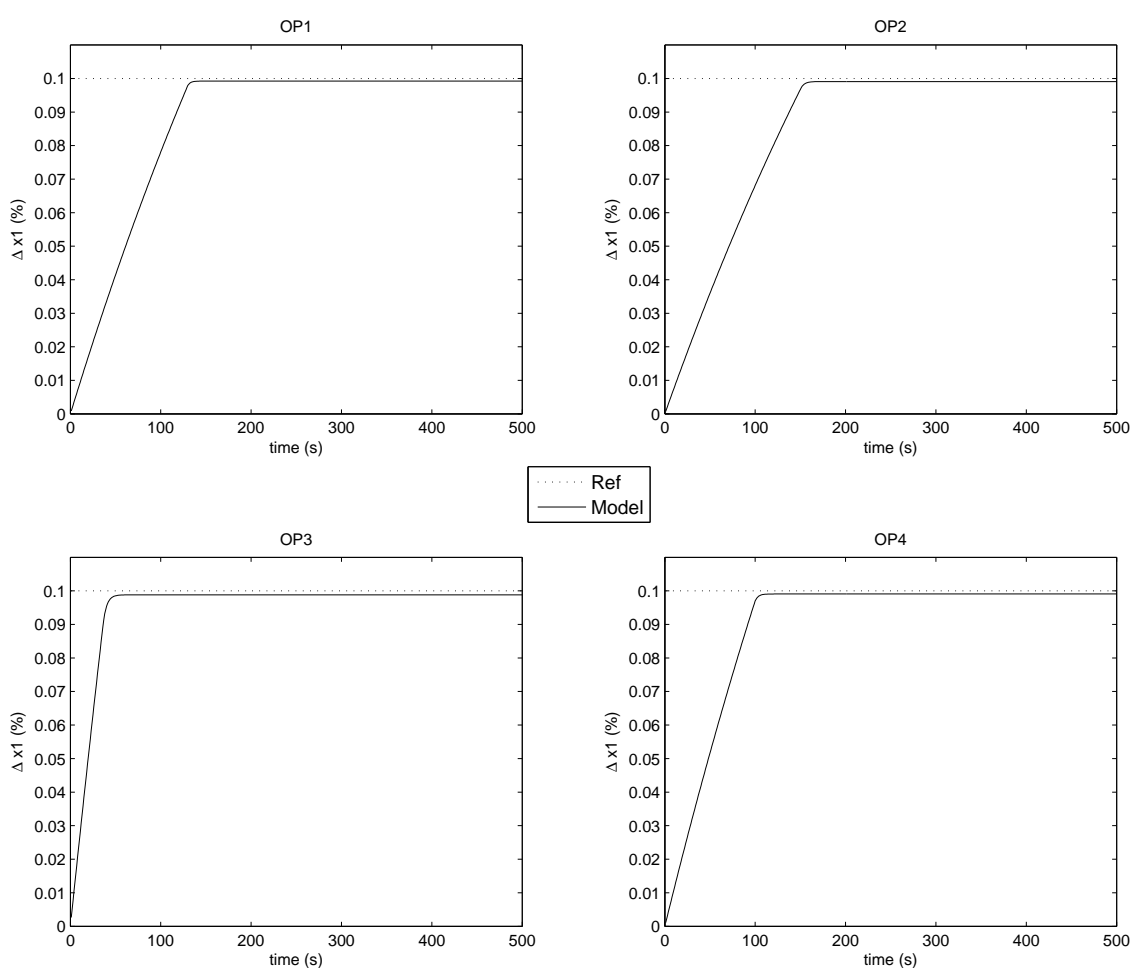

Figure 6: Control of molar fraction $x_{1}$ versus time (in seconds) for different positive and negative steps using $h_{F}$ as control variable.

It can be seen how the control (with and without perturbations) performs well but it is quite slow. The reason for this is the size of the column, described in Table 1. It can be seen how the intermediate plates are quite big while the reboiler and the reflux compartments are even bigger. This makes the control signals be effective but slow. Nevertheless the controller has shown a good performance and in a smaller column the issue of the speed of the controlled product would be avoided. 


\section{Optimization}

As shown in Figure 4 and described through all the text, the control can be performed by 3 different inputs. That allows the election of one of them attending to the chosen criteria.

These criteria could be imposed by two methods. One is the consideration of the availability of the resources needed to perform the control. The other is by the reach of each variable in the output level. For the $h_{F}$ and $q_{N}$, variables a heat source is needed, whereas a cooling source is needed for the $R$ control input case.

As well as the reach of the $q_{N}$ is the smallest one $( \pm 0.05 \%$ in the example described here) it limits the control by means of this variable. The reach of the control by the other two variables is bigger $( \pm 0.5 \%)$, allowing a big performance zone.

However, given that all the resources are available and the control could be built by any of the three control input, the selection criterion is, industrially attending, economic. As far as all the controls perform well, the cheaper one will be the chosen. Anyway, it is not only the cost of the application what should be studied, but also the benefits from production.

To apply heat to the stream feed or to the reboiler is more expensive than to subtract heat from the condenser, but the amount of product decreases when $\mathrm{R}$ input gets reduced.

The prices of fuel to get a heat source or the cooling water to perform the refrigeration must be updated from the market, as well as the price of the distillate to be sold. Therefore, not too many results can be exposed, but several conclusions can be drawn:

- When control is applied by $h_{F}$ or $q_{N}$ variables, the amount of product varies even if $R$ is not modified, but this relation is no direct.

- Control by $R$ is always the cheaper one looking at application price.

- The amount of product varies directly with $R$ changes.

- In all cases applied in the example case, control by $R$ is the most profitable one, because the amount of product is not very different but the application price is much smaller than when control is performed by the other variables. 
However, as said before, generalizations cannot be stated from these particular conclusions. In each case, the study must be performed again, due to price changes and amount of product obtained.

\section{Conclusions}

A velocity-based fuzzy control has been applied to a binary distillation simulated by a dynamic model. The necessary data were obtained using a dynamic distillation model that uses feed characteristics (enthalpy state), reflux ratio, and reboiler heat duty as inputs. The model was also useful to validate the efficiency of the fuzzy controller.

The final control structure consisted of a set of 5 local control models that were able to change the reflux ratio and heat duty either of the reboiler or of the feed stream in order to meet effectively the specified product quality for a wide range of feed variation. The results showed that the fuzzy controller was able to allow changes from a predefined output composition $x_{1}$ to another, and the controller also was capable to keep the target output in the desired range. The developed techniques are liable to be applied to more complex distillations. It was also seen that the variable to perform the control could be chosen in most of industrial cases, either by the availability of each one of the control variables or by economic reasons.

\section{Acknowledgements.}

The authors acknowledge the partial funding of this work by the projects: Regional Government Project GVPRE/2008/108, and National Projects DPI200766728-C02-01 and DPI2008-06737-C02-01.

\section{References}

[1] R. Babuska. Fuzzy Modeling and Identification. PhD thesis, Delft University of Technology, Delft, The Netherlands, 1996.

[2] Navarro J. L.; Sala A. Díez, J. L. Control por planificación de ganancia con modelos borrosos. Revista Iberoamericana de Automática e Informática Industrial, 1:32-43, 2004.

[3] Navarro J. L.; Sala A. Díez, J. L. A fuzzy clustering algorithm enhancing local model interpretability. Soft Computing - A Fusion of Foundations, Methodologies and Applications, 6, 2007. 
[4] Sala A. Navarro J. L. Díez, J. L. Target-shaped possibilistic clustering applied to local-model identification. Engineering Applications of Artificial Intelligence, 19:201-208, 2006.

[5] P. Dutta and R. R. Rhinehart. Application of neural network control to distillation and an experimental comparison with other advanced controllers. ISA Transactions 38, pages 251-278, 1999.

[6] S. Grüner, K-D. Mohl, A. Kienle, D. Gilles, G. Fernholz, and M. Friedrich. Nonlinear control of a reactive distillation column. Control Engineering Practice, 11(8):915-925, 2003.

[7] Leithead W. E. Leith D. J. Survey of gain scheduling analysis and design. International Journal of Control, 73:1001-1025, 2000.

[8] Floudas C.A. Luyben M.L. Analyzing the interaction of design and control .1. a multiobjective framework and application to binary distillation synthesis. Computers \&6 Chemical Engineering, 18:933-969, 1994.

[9] Dahule R.K. Ghosh S. Rahman I. Tambe S.S. et al. Ramanathan S.P., Mukherjee S. Optimization of continuous distillation columns using stochastic optimization approaches. Chemical Engineering Research 8 Design, 73:310-322, 2001.

[10] S. Ramchandran and R.R. Rhinehart. A very simple structure for neural network control of distillation. Journal of Process Control, 5(2):115-128, 1995.

[11] Michelsen M.L. Skovborg P. A flexible algorithm for simulation and optimization of continuous distillation. Computers \& Chemical Engineering, 16:255-262, 1992.

[12] R. E. Treybal. Mass transfer operations, 3rd edition. McGraw Hill, 1981.

[13] L. X. Wang. A Course in Fuzzy Systems and Control. Prentice-Hall. New Jersey, USA., 1997. 

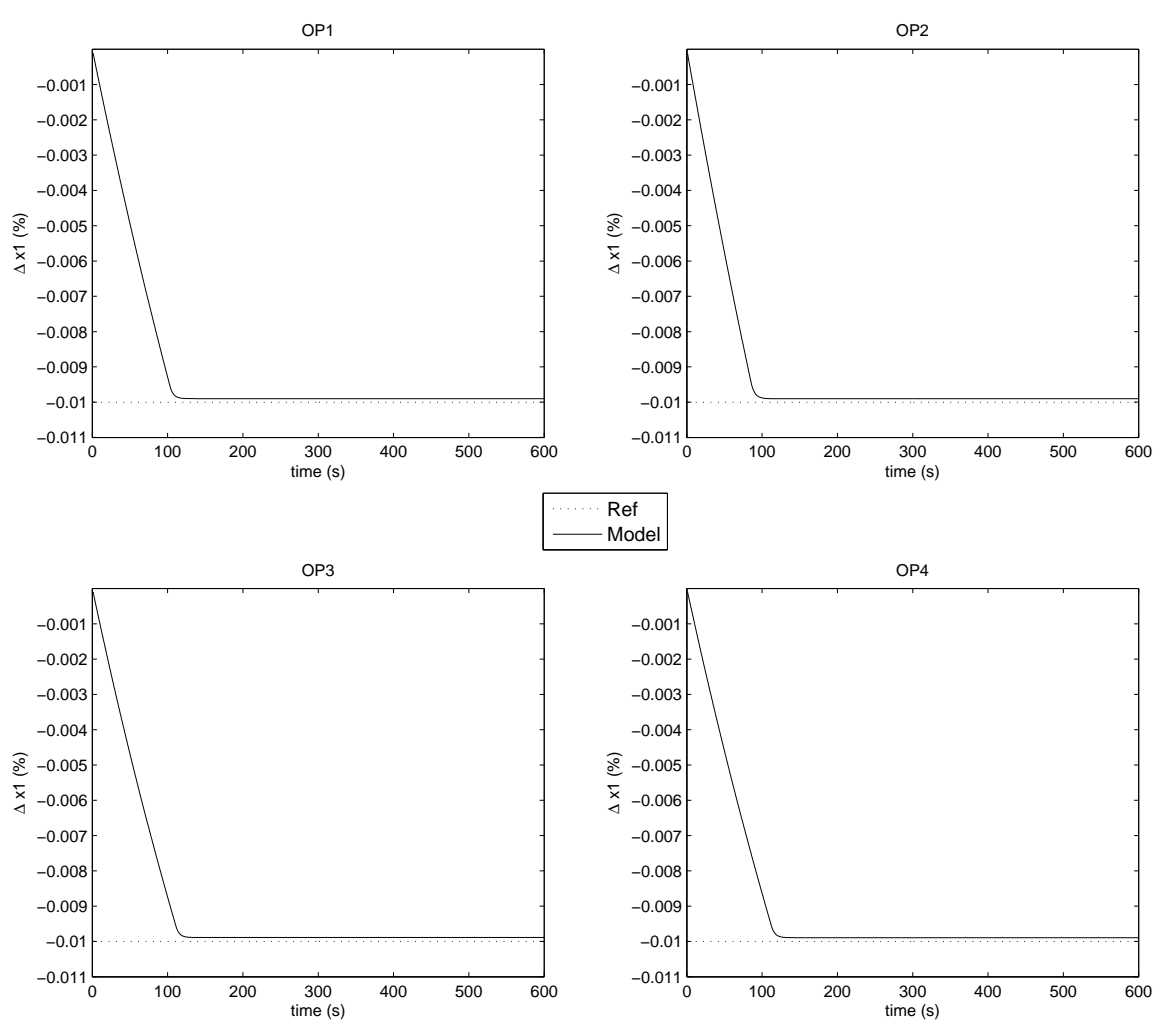

Figure 7: Control of molar fraction $x_{1}$ versus time (in seconds) for different positive and negative steps using $q_{N}$ as control variable. 

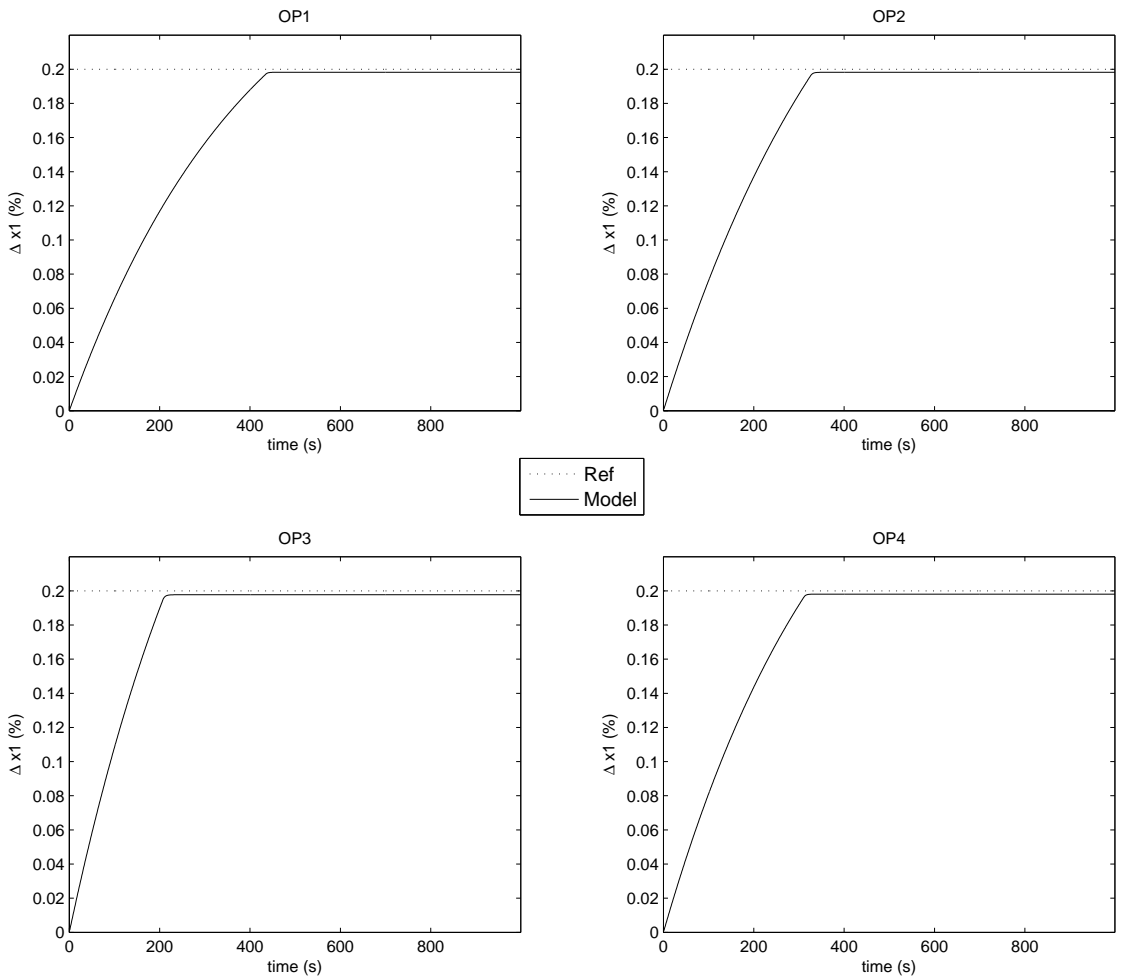

Figure 8: Control of molar fraction $x_{1}$ versus time (in seconds) for different positive and negative steps using $R$ as control variable. 


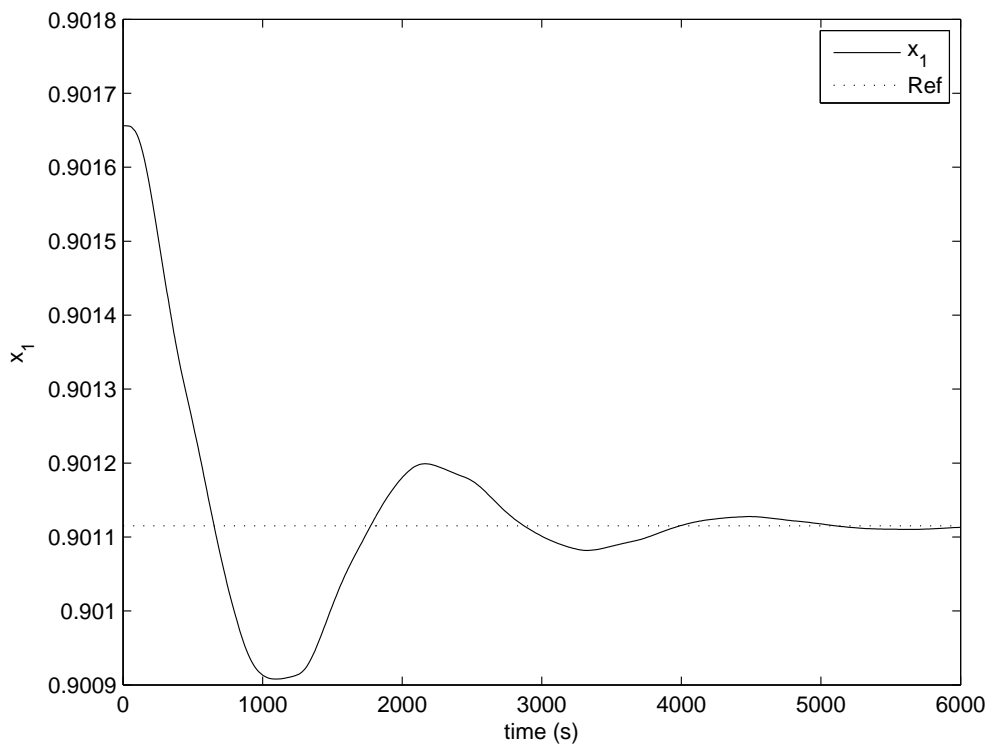

Figure 9: Control of molar fraction $x_{1}$ versus time (in seconds) for simulator using $q_{N}$. System with no perturbations 


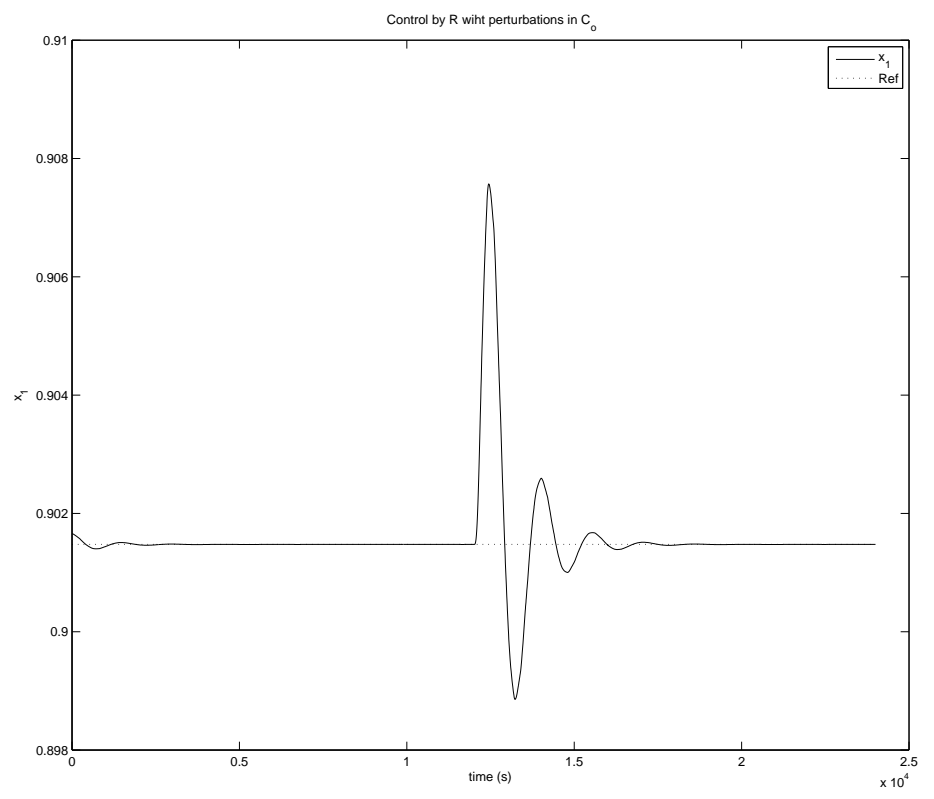

Figure 10: Control of molar fraction $x_{1}$ versus time (in seconds) for simulator using $R$. System with a perturbation in $C_{o}$ at $t=12000$ 